\title{
Performance of an Electron Beam Recorder for Disk Mastering
}

\author{
Masahiro Katsumura, Yasuo Hosoda, Hiroshi Nishiwaki, Kazuhiro Yamato *, Takanobu Higuchi, \\ Yoshiaki Kojima, Yasumitsu Wada, Tetsuya Iida and Fumihiko Yokogawa
}

\begin{abstract}
Advanced Disk System Department, PIONEER Corporation, 6-1-1, Fujimi, Tsurugashima Ccity, Saitama, 350-2288, Japan *Optical Technical Center, PIONEER Corporation, 465 Osato, Kofu City, Yamanashi, 400-0053, Japan
\end{abstract}

\begin{abstract}
We have been developing an electron beam recorder (EBR) for high-density optical disk mastering, and use it for read-only memory (ROM) and rewritable (RW) substrate mastering. We confirmed a $4.4 \%$ jitter value at 25 GB ROM disk. The reproducing characteristics of the high-density optical disk were dominated by the signal-to-noise ratio and the cross-talk. We fabricated an RW substrate with a $320-\mathrm{nm}$ track pitch and confirmed a $5.4 \%$ jitter value for a data bit length of $112 \mathrm{~nm}$ after 100 direct-over-write and crosswrite cycles.
\end{abstract}

Key words: electron beam, mastering, high-density recording, optical disk

\section{Introduction}

A user data capacity of more than $23 \mathrm{~GB}$ is required for next-generation optical disk to record high definition digital television (HDTV) broadcasting. High numerical aperture (NA) objective lenses and blue-violet semiconductor lasers have been developed to realize large capacity optical disks. In the development of nextgeneration optical disk systems, new technologies are improving steadily and several systems have been proposed. In the mastering, the track pitch and the minimum pit length become narrower and shorter, respectively, to achieve large capacity. Thus the mastering recorder is required to have high-resolution performance. To achieve high-density recording, a photo-bleachable dye in the laser beam recorder (LBR $)^{1}$, a deep-ultraviolet ray $\mathrm{LBR}^{2)}$ and a solid immersion lenses that enlarges the NA of a lens ${ }^{3}$ have been developed. Furthermore, aiming at much higher density, there were works that applied nearfield optics technology $y^{4}$. In accordance with this trend forward high-density recording, we have been developing an electron beam recorder $(\mathrm{EBR})^{5}$ ) that has high resolution performance.

Here we report the outline and basic performance of our EBR, and explain the reproducing characteristics of over 25 GB ROM disks. A plasma etching technique was adopted for groove recording disk mastering to avoid the influence of electron scattering. After describing the characteristics of a 25 GB RW disk, we report a possibility of achieving high-speed recording by adopting a chemically amplified resist.

\section{EBR structures and beam adjustment}

\section{2-1.Outline of the EBR}

Figure 1 shows a schematic picture of the EBR. A work chamber was made of iron plates in order to shield the electron beam from fluctuations of the environmental magnetic field. This EBR was a prototype made in 1993. As it has no load-lock chamber, the substrate is exchanged manually through an exchange window that can be opened or closed manually.

A cross-roller stage (X-stage) is moved in the $\mathrm{X}$ direction by a dc motor and a lead screw. A spindle motor

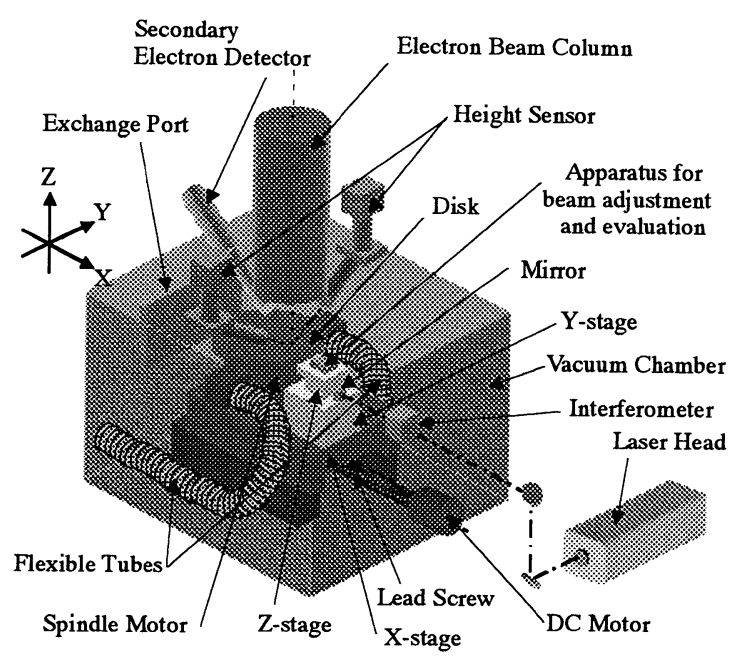

Fig. 1 Schematic picture of our EBR 
is mounted on the top of the X-stage, and is connected to the outside of the work chamber by two flexible tubes. These are connected to the inside of the spindle motor and to the external atmosphere. One flexible tube is used to carry a compressed-air tube for air bearing, a water tube for cooling and an electric cable for the motor drive. The other tube is used to release the air evolved from the air bearing. At adjacent position to the spindle motor, there are apparatuses of the electron beam adjustment on an Y$\mathrm{Z}$ stage.

An electron beam column is mounted close to the center of the top of the work chamber. A secondary electron detector is placed at one side of the column, and the illumination part and detection part of a height sensor are located opposite at another side. Although not shown in Fig.1, the work chamber is mounted on a table floated by air isolators, and vacuum components are suspended from the table.

\section{2-2. Electron beam column}

Figure 2 shows a schematic diagram of the electron beam column. The emitter is a thermal-field type ${ }^{\circ}$ that is commercially available. The first lens (condenser lens) consists of an extractor, a focus electrode and a ground electrode. It converges and accelerates the electrons simultaneously. The electron beam forms a crossover point at a middle of the blanking plates. The electron beam is limited in its angle with the diameter of the objective aperture, and propagates into an objective lens. The electron beam is focused onto the surface of a disk by adjusting the power of the objective lens according to the disk height.

In the optical disk mastering, there are several important items in the electron column. The several components in the column are used for the disk mastering.

For the ROM disk mastering, beam modulation is necessary to record the pits, and it is carried out by the blanking plates and the objective aperture. When a voltage is applied to the blanking plates for the beam modulation, the beam is deflected and intercepted by the objective aperture. The deflection pivot point coincides with the center of the blanking plates. Therefore it is important that the beam forms the crossover point to the middle of the blanking plates for high accuracy recording.

For RW disk mastering, beam wobbling is needed to record the address of the groove. The beam wobbling is done by using the deflection coil.

A focus lens, an alignment coil and the blanking plates are used during recording. The focus lens is a coreless type with a sensitivity of $11 \mu \mathrm{m} / \mathrm{mA}$ and a frequency limit of about $100 \mathrm{kHz}$. The sensitivity of the alignment coil is $1.1 \mu \mathrm{m} / \mathrm{mA}$ and the frequency limit is 70 $\mathrm{kHz}$. The beam blanking time is $10 \mathrm{nsec}$.

\section{2-3. Beam adjustment and evaluation}

The beam adjustment is an important item for accurate recording. In the disk mastering, it is not possible to adjust the beam by focusing on the resist substrate, thus

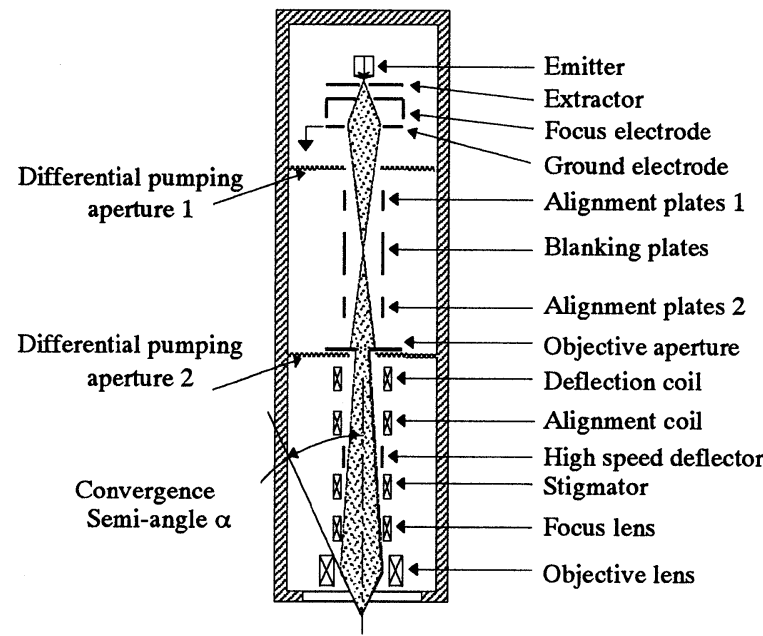

Fig. 2 Schematic diagram of the electron beam column.

we use a reference sample and a Faraday cup with a knife-edge on it to measure the beam diameter. First we measure the disk height using the optical height sensor, and set the reference sample to the same position by adjusting the $\mathrm{Z}$-stage position. The reference sample is composed of a silicon wafer fragment that is inlaid with latex balls whose diameters range from 0.1 to $0.5 \mu \mathrm{m}$ and finally coated with platinum by sputtering. We adjust the focal distance of the objective lens and a stigmator to obtain the highest resolution scanning electron microscope (SEM) image of the latex balls. After the adjustment is completed, we set the Faraday cup to the same height of the reference sample, and measure the beam current using the Faraday cup, and measure the diameter using the knifeedge method. The beam diameter and current have a close relation to the disk reproducing performance.

\section{Characteristics of the EBR}

\section{3-1. Beam size and beam current}

The beam diameter can be changed by selecting the size of the objective aperture. The measured and calculated beam currents and beam diameters at convergence semiangles of $3 \mathrm{mrad}$ and $6 \mathrm{mrad}$ are listed in Table $\mathrm{I}$. The measured beam diameters are slightly larger than the calculated values. We estimated that one of the factors causing the difference is the astigmatism of the beam.

To evaluate the focal depth of the beam at a $50 \mathrm{keV}$ beam energy, we recorded some patterns by changing the recording surface height. In the experiment, we recorded some patterns for the purpose of beam profile observation.

Figure 3 shows the pit patterns when the recording surface height is shifted to about $\pm 10 \mu \mathrm{m} 0$-p. The beam astigmatism is clearly observed in this figure. From these results, we confirmed the focal depth of the beam was within $\pm 10 \mu \mathrm{m} 0-\mathrm{p}$.

On the other hand, in our past experiment, the vertical disk surface run-out was about $15 \mu \mathrm{m} 0-\mathrm{p}$, which is larger than the focal depth. To compensate for the defocusing by the run-out, we adopted a focusing control 
method. The focus is controlled by correcting the focal distance of the focus lens according to the disk height information measured by the optical height sensor. As a result, the pit geometry remains unchanged even when the variation of the disk height is $\pm 30 \mu \mathrm{m} 0$-p.

Table I. Measured and calculated beam diameters, and beam currents for convergence semiangles of 3 mrad and 6 mrad.

\begin{tabular}{|c|c|c|c|c|}
\hline Convergence & \multicolumn{2}{|c|}{ Beam diameter [nm] } & \multicolumn{2}{c|}{ Beam current [nA] } \\
\cline { 2 - 5 } semiangle & measured & calculated & measured & calculated \\
\hline \hline $3 \mathrm{mrad}$ & 37 & 25 & 34 & 36 \\
\hline $6 \mathrm{mrad}$ & 55 & 52 & 130 & 137 \\
\hline
\end{tabular}

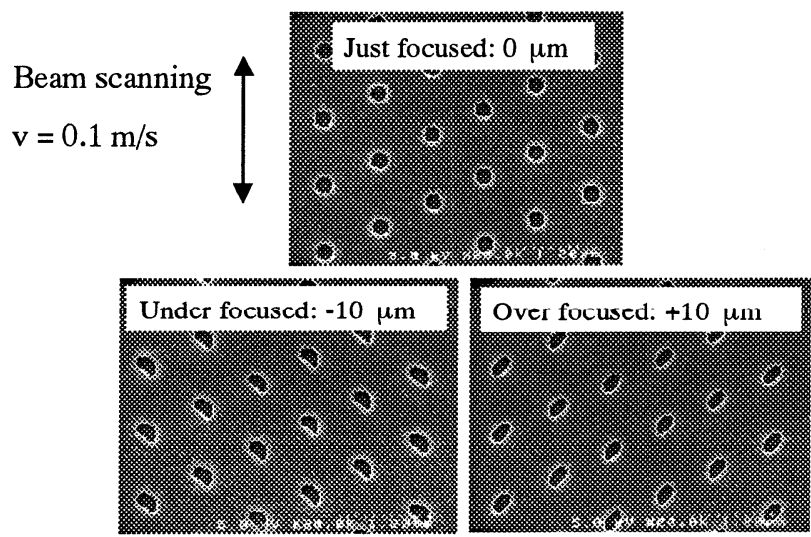

Fig. 3 Astigmatism of the focused beam when the recording surface height was shifted by about $10 \mu \mathrm{m}$.

\subsection{Deviation of the track pitch}

We recorded a groove with a $0.74 \mu \mathrm{m}$ track pitch at a $0.5 \mathrm{~m} / \mathrm{s}$ recording velocity to evaluate the track pitch deviation. We fabricated a stamper from this master disk and used the stamper as a measurement specimen with an enough reflection ratio, and measured the track pitch using a laser-scanning microscope. We recorded a wide groove at a broad track pitch, because the laser-scanning microscope had a resolution limit. The deviation of the track pitch was a $28.5 \mathrm{~nm}$ peak-to-peak and the standard deviation was a $2.9 \mathrm{~nm}$ as shown in Fig. 4. We presumed that there are several factors responsible for the track pitch deviation such as the beam displacement, a non-repeatable run-out of the spindle motor and an error in the beam position correction. These factors will be one of the subjects of our next study.

\section{Resolution performance of our EBR}

We recorded various patterns to evaluate the resolution performance of our EBR. First we changed the convergence semiangle to verify the convergent capability of the electron beam. We adjusted the convergence semiangles to $3 \mathrm{mrad}$ and $6 \mathrm{mrad}$, and observed the beam characteristics shown in Table I. We recorded a groove and a random signal with these conditions, and observed

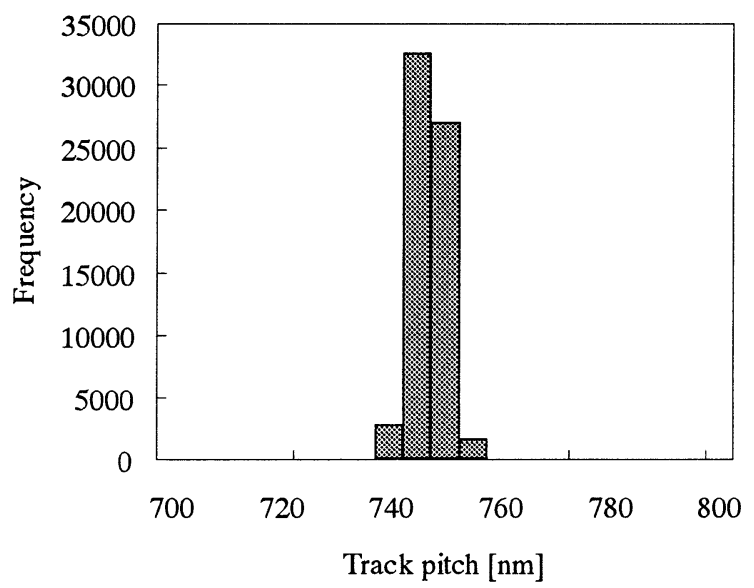

Fig. 4 Deviation of the track pitch.

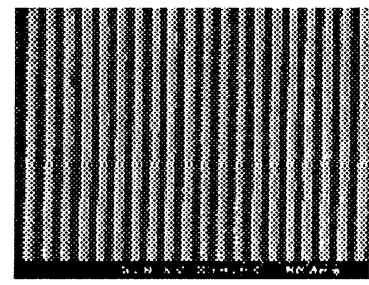

(a) $80 \mathrm{~nm} \mathrm{L \& S} \mathrm{(6} \mathrm{mrad)}$

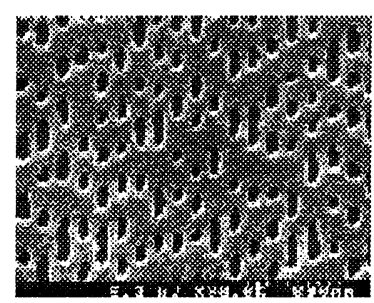

(c) $75 \mathrm{~GB}(6 \mathrm{mrad})$

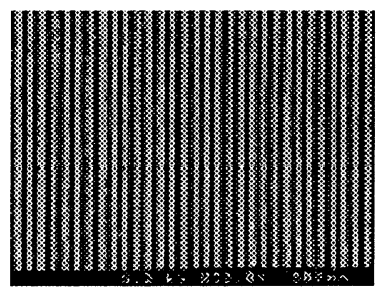

(b) $50 \mathrm{~nm}$ L\&S (3 mrad)

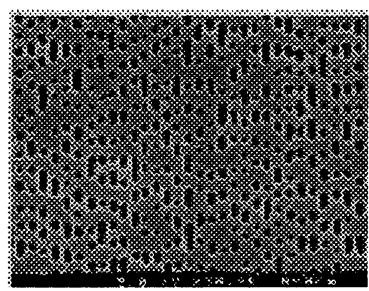

(d) $201 \mathrm{~GB}(3 \mathrm{mrad})$
Fig. 5 Experimental resolution limit for (a) a 6 mrad convergence semiangle and (b) a $3 \mathrm{mrad}$ convergence semiangle. There is a possibility of huge-capacity recording with 8-16 modulation for future-generation optical disks.

the resist patterns by SEM. Figure 5(a) shows an $80 \mathrm{~nm}$ line-and-space pattern that is an experimental resolution limit of our EBR at $6 \mathrm{mrad}$. Figure 5(b) shows $50 \mathrm{~nm}$ line-and-space that is an experimental resolution limit at 3 mrad. Figures 5(c) and 5(d) show the pit patterns recorded in each condition. They correspond to recording capacities of $75 \mathrm{~GB}$ and $201 \mathrm{~GB}$, respectively, calculated from the DVD format condition.

The above were only observations of the pattern and the beam adjustment for high-density recording will be necessary in future. We verified the resolution performance of our EBR by observing the resist pattern and confirmed the ability to realize mastering for further generation optical disks. 


\section{ROM experiment and results}

The recording capacity depends on the track pitch, the minimum pit length, the modulation method, and the format efficiency. We selected 17PP modulation as the modulation method with a format efficiency of $81.74 \%$, and examined the optimal disk parameters. To optimize the parameters for a high-density optical disk, we changed the track pitch and the minimum pit length. In this experiment, we chose the recording capacity of $25,27.5$ and $30 \mathrm{~GB}$. Table II shows the disk parameters for each recording capacity with $17 \mathrm{PP}$ modulation. Keeping the target recording capacity, we adjusted the track pitch and the minimum pit length respectively. In this experiment, we chose track pitches from $240 \mathrm{~nm}$ to $300 \mathrm{~nm}$ in consideration of the reproducing system.

Table II. Disk parameters.

Relationship between the minimum pit length and the track pitch.

\begin{tabular}{|c|c|c|c|c|}
\hline Track pitch & 240 & 260 & 280 & 300 \\
\hline \hline 25 GB & 199 & 183 & 170 & 159 \\
\hline 27.5 GB & 180 & 167 & 155 & 145 \\
\hline 30 GB & 165 & 153 & 142 & 132 \\
\hline
\end{tabular}

We recorded a random digital signal with the parameters shown in table II. After fabricating stampers using a galvanics process, we attached a glass substrate with photo polymer, and transcribed the pattern from the stampers. We used the glass as the substrate, and formed a reflective layer and a cover layer on the substrate. The thickness of the cover layer was $100 \mu \mathrm{m}$.

To evaluate the capability of the high-density optical disks, we used a reproducing system with a blue-violet laser diode of $405 \mathrm{~nm}$ wavelength and a 0.85 NA objective lens. We adopted a combination of a conventional preequalizer and a limit equalizer ${ }^{7)}$ as the reproducing equalizer. At first, the system margin was not considered in evaluating the EBR performance. We adopted the differential phase detection method as the tracking method and the astigmatic method as the focusing method respectively.

Figure 6 shows the read-out results for each fabricated disk. In case of the $25 \mathrm{~GB}$ disk, the jitter value was improved by decreasing the track pitch from $300 \mathrm{~nm}$ and we observed a $4.4 \%$ jitter value at the $280 \mathrm{~nm}$ track pitch parameter. By decreasing the track pitch furthermore, the jitter value became worse.

The minimum pit length became longer by decreasing the track pitch in case of the fixed recording capacity. The signal-to-noise ratio (SNR) of the reproducing system improved as the minimum pit length was increased. We observed the jitter deterioration as a result of further decreasing the track pitch. The SNR was improved as the minimum pit length was increased with the track pitch reduction. The cross-talk also became larger due to the track pitch reduction. The degradation due to the cross-talk became dominant in more narrower track pitch. From these results, it is clear that the dominant factor that decided the jitter value was changed by changing the disk parameters. These phenomena also appeared in the reproduction with other capacity disks. The dominant factor in the jitter deterioration became the SNR, and the best jitter disk parameter changed as the recording capacity became larger.

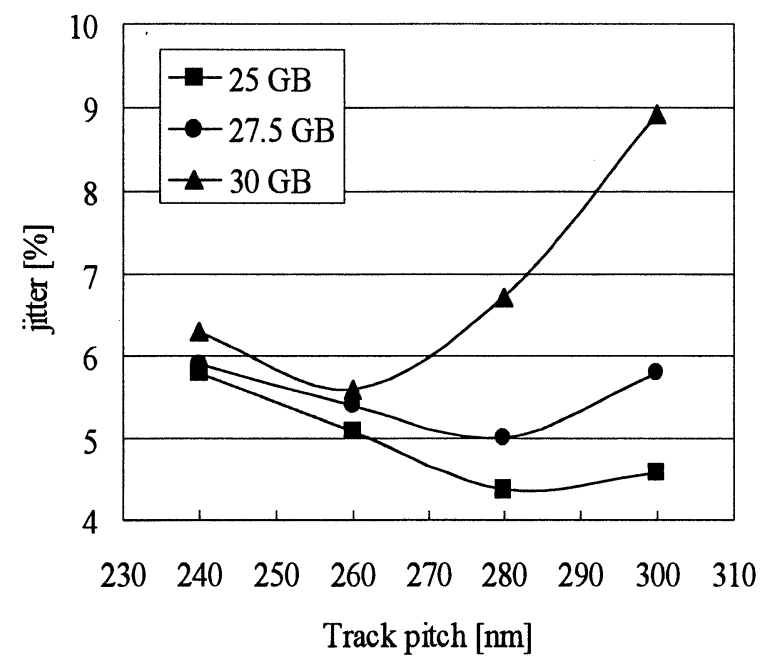

Fig. 6 Jitter values of 25, 27.5, and 30 GB disks. The equalizer condition is the combination with the conventional equalizer and the limit equalizer.

\section{6. $R W$ disk experiment and results}

The RW disk required a shallow groove depth to reduce the disk noise. To fabricate a shallow groove disk in the conventional stamper fabrication process similar to that used for ROM disks, we have to coat a thin electron beam resist. In the thin resist mastering process, the electron scattering effect appears clearly on the resist surface. To investigate the relationship between the scattering and the resist thickness, we prepared silicon wafers coated with $25 \mathrm{~nm}$ - and $70 \mathrm{~nm}$-thick resists. We then recorded the groove on each substrate and developed the groove with the developer. The groove patterns are shown in Fig. 7. As seen in the pictures, the land roughness clearly differ, and the groove became wider at the pattern of 25-nm-thick resist compare with the 70 nm-thick resist. Here we define exposed part as groove and non-exposed area between the lines as land. We estimate that the scattering electrons reached the land area, and this area was developed with the developer. The influence of the scattering is shown by the expansion of the groove width and the land roughness. It is important to consider the influence of the electron scattering and to develop a 
process that adequate for the required pattern in electron beam mastering for high-density optical disks.

Electron scattering influences cannot be avoided in electron beam recording. We estimated that the land roughness was observed as the disk noise after sputtering recording layers. On the assumption that the performance of the groove disk would deteriorate due to the disk noise, we examined the process using plasma etching technology to reduce the land roughness caused by the scattering influence ${ }^{8)}$. The resist worked as a mask in the plasma etching process. After the etching had been completed to the required groove depth, we removed the resist. The silicon wafer surface formed the land surface, and consequently the land roughness was expected to be improved. Figure 8 shows the observed result for $25-\mathrm{nm}$ thick groove created by using the plasma etching technique. The land roughness was clearly smoother.

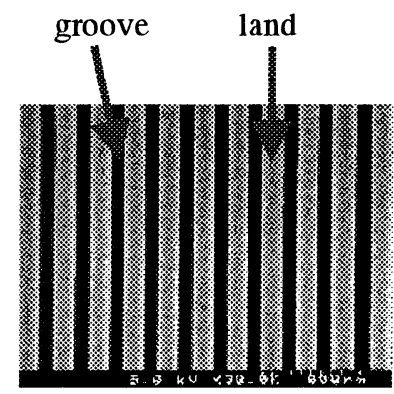

(a)

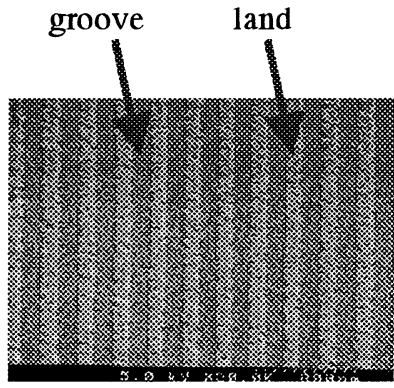

( b)
Fig. 7 Groove pattern image using the conventional process: (a) resist thickness $70 \mathrm{~nm}$, (b) resist thickness $25 \mathrm{~nm}$.

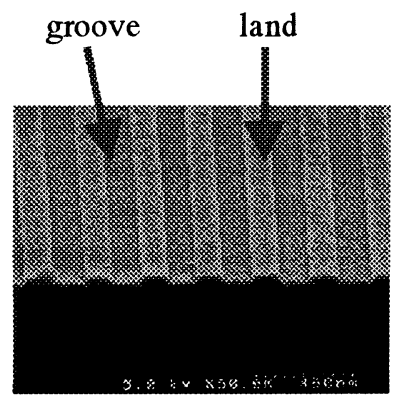

Fig. 8 Etched groove profile.

In the RW disk mastering process, we coated a 160 $\mathrm{nm}$-thick resist on a silicon wafer, exposed the groove at a 320-nm-track pitch, and developed it. After developing process, we used the resist as a mask and etched the silicon wafer to a depth of $20 \mathrm{~nm}$. The resist was removed from the silicon wafer and the stamper fabricated from it. We produced a 1.1-mm-thick disk substrate by injection molding and sputtered the several layers on it. Finally we coated the disk with a cover layer. The process time needed to etch a 20-nm-thick groove was about $60 \mathrm{sec}$ and the variation of the groove depth in the whole disk was within 2 nmp-p. Figure 9 shows a cross-sectional view of this disk

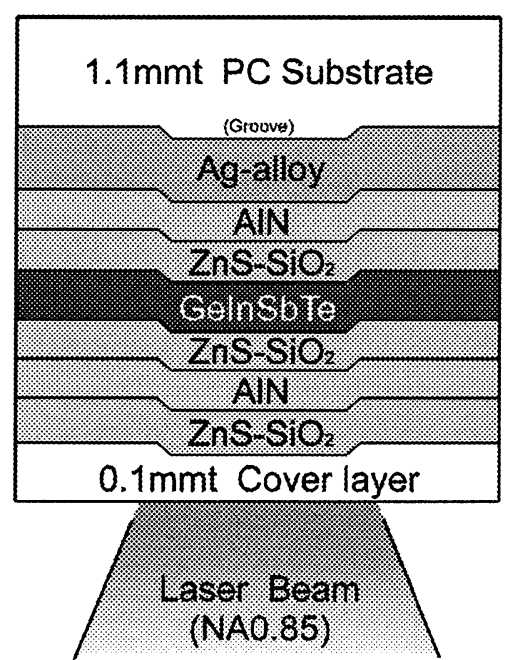

Fig. 9 Disk structure

The transparent cover layer was manufactured by using a polycarbonate sheet pasted up with a photo polymer.

Table III. Evaluating conditions and disk parameters

\begin{tabular}{|l|c|}
\hline Wavelength & $405 \mathrm{~nm}$ \\
\hline NA & 0.85 \\
\hline Disk size & $120 \mathrm{~mm}$ diameter \\
\hline Track pitch & $320 \mathrm{~nm}$ \\
\hline Groove depth & $20 \mathrm{~nm}$ \\
\hline Linear velocity & $4.92 \mathrm{~m} / \mathrm{s}$ \\
\hline Data bit length & $112 \mathrm{~nm}$ \\
\hline Equalizer & Conv.EQ / Limit EQ \\
\hline Modulation method & $17 \mathrm{PP}$ \\
\hline
\end{tabular}

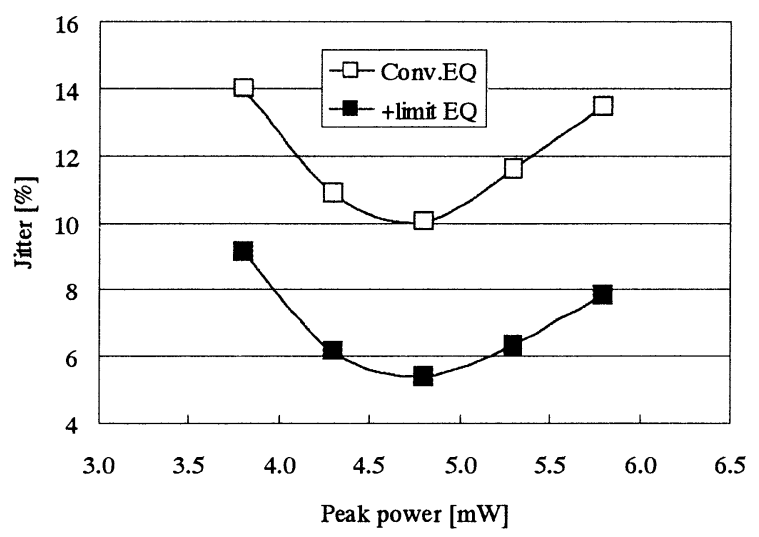

Fig. 10 Characteristics of a 25 GB RW disk and write power dependency with the additional limit EQ. (Data bit length: 112 nm, 100 DOW, and 100 $\mathrm{CW})$ 
structure and Table III shows the evaluating conditions and disk parameters.

We recorded a random data signal at a bit length of $112 \mathrm{~nm}$ with 17PP modulation. During the recording operation, the illuminating laser beam was pulsemodulated at three different power levels. The pulse width was adjustable depending on the individual mark length. Figure 10 shows the experimental results of the write power dependency with the conventional equalizer (Conv. EQ) and an additional limit equalizer (+ limit EQ) after 100 direct-overwrite (DOW) cycles and 100 crosswrite (CW) cycles. The limit EQ improved the signal-to-noise ratio of the short pit length, which resulted in a jitter improvement. We obtained a jitter value of $5.4 \%$ at a 112 $\mathrm{nm}$ bit length and realized a sufficient write power margin after $100 \mathrm{CW}$.

\section{High-speed recording}

We confirmed the EBR has sufficient performance for the high-density optical disks. However the recording speed is inadequate since it takes a long time to record the entire disk. Thus we cannot expect sufficient throughput for mass production. Therefore we tried to record faster than in the conventional process. As one method to improve the recording time, we chose a high sensitivity resist of a chemically amplified type and tried to evaluate the possibility of high-speed recording. We recorded groove by using a chemically amplified resist and evaluated the resist sensitivity by observing the groove width. Figure 11(a) shows a groove recorded at $70 \mu \mathrm{C} / \mathrm{cm} 2$ with the conventional resist process, while Figures $11(\mathrm{~b})$, $11(c)$ and $11(d)$ show grooves at $16.7 \mu \mathrm{C} / \mathrm{cm} 2$ with the

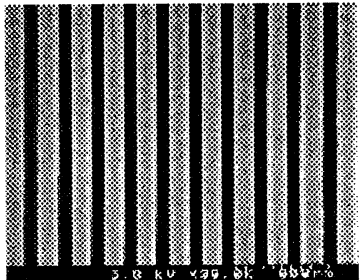

(a) Conventional process

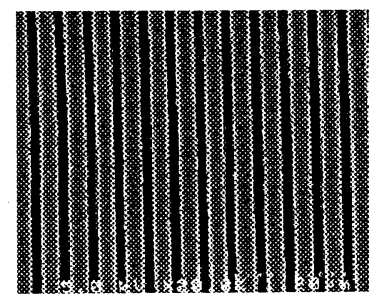

(c) Developer B

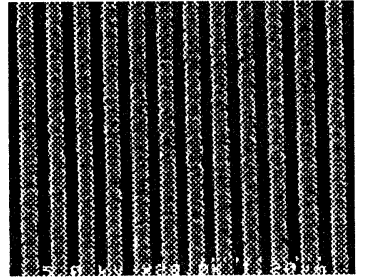

(b) Developer A

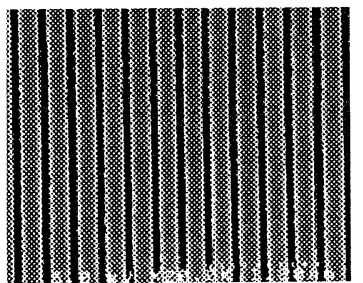

(d) Developer C
Fig. 11 High speed recording using a chemically amplified resist: (a) conventional resist at $70 \mu \mathrm{C} / \mathrm{cm}^{2}$, (b),(c) and (d) chemically amplified resist at $16.7 \mu \mathrm{C} / \mathrm{cm}^{2}$ using several developers. chemically amplified resist process made by changing developers. The surface roughness and groove width of the chemically amplified groove patterns differed widely as a result of changing the developers. These results show that the developing power influenced the resist sensitivity. The groove width of the developer A was approximately equal to the conventional resist groove width. We confirmed the possibility of more than 4 times higher speed recording using the chemically amplified resist.

\section{Conclusions}

We have developed an EBR for next-generation optical disk mastering. We confirmed that it has experimental resolution limits of $80 \mathrm{~nm}$ and $50 \mathrm{~nm}$ at convergence semiangles of $6 \mathrm{mrad}$ and $3 \mathrm{mrad}$, respectively. We also confirmed the possibility of further generation optical disks by observing 75 GB and 201 GB capacity recording. As the reproducing results, in case of a 25 GB ROM disk, we confirmed a $4.4 \%$ jitter value with the additional limit EQ. To avoid the influence of electron scattering, we chose the plasma etching process in the RW disk mastering. We fabricated a 320-nm-track pitch substrate and confirmed a $5.4 \%$ jitter value at a data pit length of $112 \mathrm{~nm}$ after $100 \mathrm{DOW}$ and $100 \mathrm{CW}$ by adjusting the writing power. We confirmed the possibility of high speed recording by using a chemically amplified resist.

\section{References}

1) T. Higuchi et al., Joint MORIS/ISOM 1997 Technical Digest

2) M. Takeda et al., Joint ISOM/ODS 1999 Technical Digest

3) S. Imanishi et al., Joint ISOM/ODS 1999 Technical Digest

4) H. J. Mamin. et al., Joint ISOM/ODS 1996 Technical Digest

5) Y. Kojima et al., Jpn. J. Appl. Phys., Vol. 37 (1998), pp. 2137-2143

6) B. E. Koek et al., Jpn. J. Appl. Phys., Vol. 32(1993), 5982

7) S. Miyanabe et al., Jpn. J. Appl. Phys., Vol. 38(1999), pp. 1715-1719

8) M. Katsumura et al., Jpn. J. Appl. Phys., Vol. 41(2002), pp. $1698-1703$

Received March 29, 2002; Accepted July 15, 2002 